\title{
Strain relaxation of GaAs/Ge crystals on patterned Si substrates
}

\author{
A. G. Taboada, ${ }^{1, a)}$ T. Kreiliger, ${ }^{1}$ C. V. Falub, ${ }^{1, b)}$ F. Isa, ${ }^{2}$ M. Salvalaglio, ${ }^{3}$ L. Wewior, ${ }^{4}$ \\ D. Fuster, ${ }^{4}$ M. Richter, ${ }^{5}$ E. Uccelli, ${ }^{5}$ P. Niedermann, ${ }^{6}$ A. Neels, ${ }^{6}$ F. Mancarella, ${ }^{7}$ B. Alén, ${ }^{4}$ \\ L. Miglio, ${ }^{3}$ A. Dommann, ${ }^{6}$ G. Isella, ${ }^{2}$ and H. von Känel ${ }^{1}$ \\ ${ }^{1}$ Laboratory for Solid State Physics, ETH Zürich, Otto-Stern-Weg 1, CH-8093 Zürich, Switzerland \\ ${ }^{2}$ L-NESS, Department of Physics, Politecnico di Milano, via Anzani 42, I-22100 Como, Italy \\ ${ }^{3}$ L-NESS, Department of Materials Science, Università di Milano-Bicocca, via Cozzi 55, I-20125 Milano, Italy \\ ${ }^{4}$ IMM, Instituto de Microelectrónica de Madrid (CNM, CSIC), C/Isaac Newton 8, E-28760 Tres Cantos, \\ Madrid, Spain \\ ${ }^{5}$ Functional Materials Group, IBM Research-Zürich, Säumerstrasse 4, CH-8803 Rüschlikon, Switzerland \\ ${ }^{6}$ Centre Suisse d'Electronique et Microtechnique, Jaquet-Droz 1, CH-2002 Neuchatel, Switzerland \\ ${ }^{7}$ CNR-IMM of Bologna, Via Gobetti 101, I-40129 Bologna, Italy
}

(Received 19 November 2013; accepted 28 December 2013; published online 16 January 2014)

\begin{abstract}
We report on the mask-less integration of GaAs crystals several microns in size on patterned $\mathrm{Si}$ substrates by metal organic vapor phase epitaxy. The lattice parameter mismatch is bridged by first growing $2-\mu \mathrm{m}$-tall intermediate Ge mesas on $8-\mu \mathrm{m}$-tall Si pillars by low-energy plasma enhanced chemical vapor deposition. We investigate the morphological evolution of the GaAs crystals towards full pyramids exhibiting energetically stable $\{111\}$ facets with decreasing Si pillar size. The release of the strain induced by the mismatch of thermal expansion coefficients in the GaAs crystals has been studied by X-ray diffraction and photoluminescence measurements. The strain release mechanism is discussed within the framework of linear elasticity theory by Finite Element Method simulations, based on realistic geometries extracted from scanning electron microscopy images. (C) 2014 AIP Publishing LLC. [http://dx.doi.org/10.1063/1.4861864]
\end{abstract}

The monolithic integration of III-V semiconductors providing optoelectronic functionalities to the mainstream $\mathrm{Si}$ based microelectronics technology is one of the keys to future multifunctional semiconductor devices. It is challenging to realize, however, because of defects caused by the mismatch of lattice parameters and thermal expansion coefficients. For GaAs on $\mathrm{Si}$ the lattice and thermal mismatch amounts to $4.1 \%$ and $120.4 \%$, respectively, at $300 \mathrm{~K}$. Antiphase domains (APDs) are additional frequently present defects. ${ }^{1}$

Previous approaches for the integration of GaAs on $\mathrm{Si}$ included low temperature nucleation by metal organic vapor phase epitaxy (MOVPE) $)^{2,3}$ and molecular beam epitaxy, ${ }^{4-6}$ the use of Ge or GeSi virtual substrates, ${ }^{7-11}$ and the incorporation of intermediate graded GaAsP or InGaP layers. ${ }^{12}$

Substrate patterning offers additional benefits because most threading dislocations can escape through the sidewalls of crystals exhibiting sufficiently large aspect ratios (height/width). ${ }^{13}$ A similar mechanism coined aspect ratio trapping prevails when $\mathrm{GaAs}$ and $\mathrm{GaAs} / \mathrm{Ge}$ are grown into submicron sized openings of dielectric masks. ${ }^{14-16}$ Even vertical dislocations perpendicular to the substrate surface are expelled from finite-sized crystals by interacting with slanting facets. ${ }^{17,18}$

The recently reported method of three-dimensional (3D) heteroepitaxy, providing space filling arrays of tall, relaxed Ge crystals on patterned $\mathrm{Si}(001)$ substrates, may emerge as a promising solution also for the integration of $\mathrm{GaAs}$ on $\mathrm{Si}^{19}$

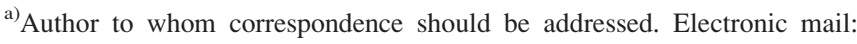
gonzalez@phys.ethz.ch.

b) Present address: OC Oerlikon Advanced Technologies, Iramali 18, 9496 Balzers, Liechtenstein.
}

Using these Ge crystal arrays as seeds for GaAs would be advantageous, since GaAs and Ge are almost perfectly lattice matched.

In this work, we report on the MOVPE growth of strainfree GaAs crystals on Si substrates patterned at the micron scale. Nominal $\mathrm{Si}(001)$ and offcut $\mathrm{Si}(001)$ substrates $\left(6^{\circ}\right.$ towards [110]) were patterned by conventional photolithography and deep reactive ion etching (DRIE) based on the Bosch process. ${ }^{20}$ The patterns consisted of $8-\mu \mathrm{m}$ tall pillars, spaced uniformly by gaps of 3 and $4 \mu \mathrm{m}$, or in blocks of $10 \times 10$ separated by $5-\mu$ m-wide trenches. The $\mathrm{Si}$ pillar width ranged from $2 \mu \mathrm{m}$ to $40 \mu \mathrm{m}$. Two micron tall Ge mesas were first grown by low-energy plasma enhanced chemical vapor deposition (LEPECVD) at $\mathrm{T}=500^{\circ} \mathrm{C}$ and a growth rate of $4.2 \mathrm{~nm} / \mathrm{s}$ and cyclically annealed between $600^{\circ} \mathrm{C}$ and $780^{\circ} \mathrm{C}^{21}$ The widely used two-step method was employed for the GaAs growth by MOVPE. ${ }^{22}$ After a $7 \mathrm{~nm}$ thick seed layer deposited at $\mathrm{T}=500^{\circ} \mathrm{C}, \mathrm{GaAs}$ crystals between 2 and $4 \mu \mathrm{m}$ in height were grown onto the $\mathrm{Ge} / \mathrm{Si}$ mesas at $\mathrm{T}=680^{\circ} \mathrm{C}$ at a growth rate of $28 \mathrm{~nm} / \mathrm{min}$ and a reactor pressure of 100 millibars. GaAs grown on offcut planar $\mathrm{Ge} / \mathrm{Si}$ substrates and on semi-insulating $\mathrm{GaAs}(001)$ substrates served as control samples. The morphology of the $\mathrm{GaAs} / \mathrm{Ge} / \mathrm{Si}$ crystals was characterized with a Zeiss ULTRA 55 scanning electron microscope (SEM). Cross sections were obtained by focused ion beam (FIB) milling in a Zeiss NVision 40 dual beam FIB/SEM system. High resolution X-ray diffraction (HRXRD) was performed with $\mathrm{Cu} K \alpha_{1}$ radiation using a PANalytical X'Pert Pro-MRD diffractometer equipped with a 4-bounce $\mathrm{Ge}(220)$ crystal monochromator on the incident beam, and an analyzer crystal and a Xe point detector on the diffracted beam. The scattering plane was chosen perpendicular to the offcut direction. A $532 \mathrm{~nm}$ 
continuous wave laser line was used to excite the photoluminescence (PL) at $\mathrm{T}=5 \mathrm{~K}$. The PL spectrum was recorded with an InGaAs line detector attached to a $0.3 \mathrm{~m}$ focal length spectrograph. Micro-PL measurements were done at $4 \mathrm{~K}$ within a low vibration Attocube Attodry 1000 cryostat, with a $785 \mathrm{~nm}$ laser diode for excitation and a low noise silicon charge coupled device for detection. Elasticity-theory calculations by Finite Element Method (FEM) simulations with the COMSOL Multiphysics solver were performed to evaluate the effect of patterning on the thermal strain. Experimental geometries were approximated by $3 \mathrm{D}$-crystals with $\mathrm{C} 4$ symmetry and based on top- and cross-sectional SEM images.

SEM images of typical GaAs/Ge crystals on Si pillars are shown in Fig. 1. For the narrowest, $2 \mu \mathrm{m}$ wide pillars, they have a pyramidal shape with well-defined $\{111\}$ facets (Figs. 1(a), 1(c)-1(f)). Crystals grown on wider pillars exhibit a more complicated morphology with additional facets as exemplified in Figs. 1(b), 1(g)-1(i) for a pillar width of $15 \mu \mathrm{m}$. The wafer patterning prevents thermal crack formation, which is present at a density of $\delta_{\text {Cracks }}=7.8 \pm 2.3 \mathrm{~mm}^{-1}$ along the offcut direction in the planar GaAs/Ge/Si control samples. ${ }^{23}$ In contrast to the symmetric appearance of GaAs pyramids grown on nominal substrates (Figs. 1(a)-1(e)), those on the vicinal ones (Figs. 1(c) and 1(e)) are tilted towards the [001] direction of the substrate. The tilt angle of $6^{\circ}$ corresponds to the offcut angle of the Si substrate. Larger GaAs structures exhibit the same kind of asymmetry along [110] (Figs. 1(b), 1(g)-1(i)), while 2- $\mu$ m-thick Ge mesas preserve the substrate miscut. ${ }^{17}$ The asymmetrical distribution of $\{111\},\{113\}$ facets, and the top (001) facet results both from the substrate miscut of $6^{\circ}$ and the coexistence of $\operatorname{GaAs}\{111\} \mathrm{A}$ and $\operatorname{GaAs}\{111\} \mathrm{B}$ facets. The $\{111\} \mathrm{B}$ facet is very stable, resulting in a low growth rate, especially compared with the rates of the top (001) facet and the $\{111\} \mathrm{A}$ facets. ${ }^{24}$ This asymmetry is especially evident comparing the (110) and (1-10) FIB cross sections of Figs. 1(h) and 1(i). Along the miscut direction, just a small part of the surface remains parallel to the offcut substrate plane, while the exact [001] surface orientation is almost fully restored.
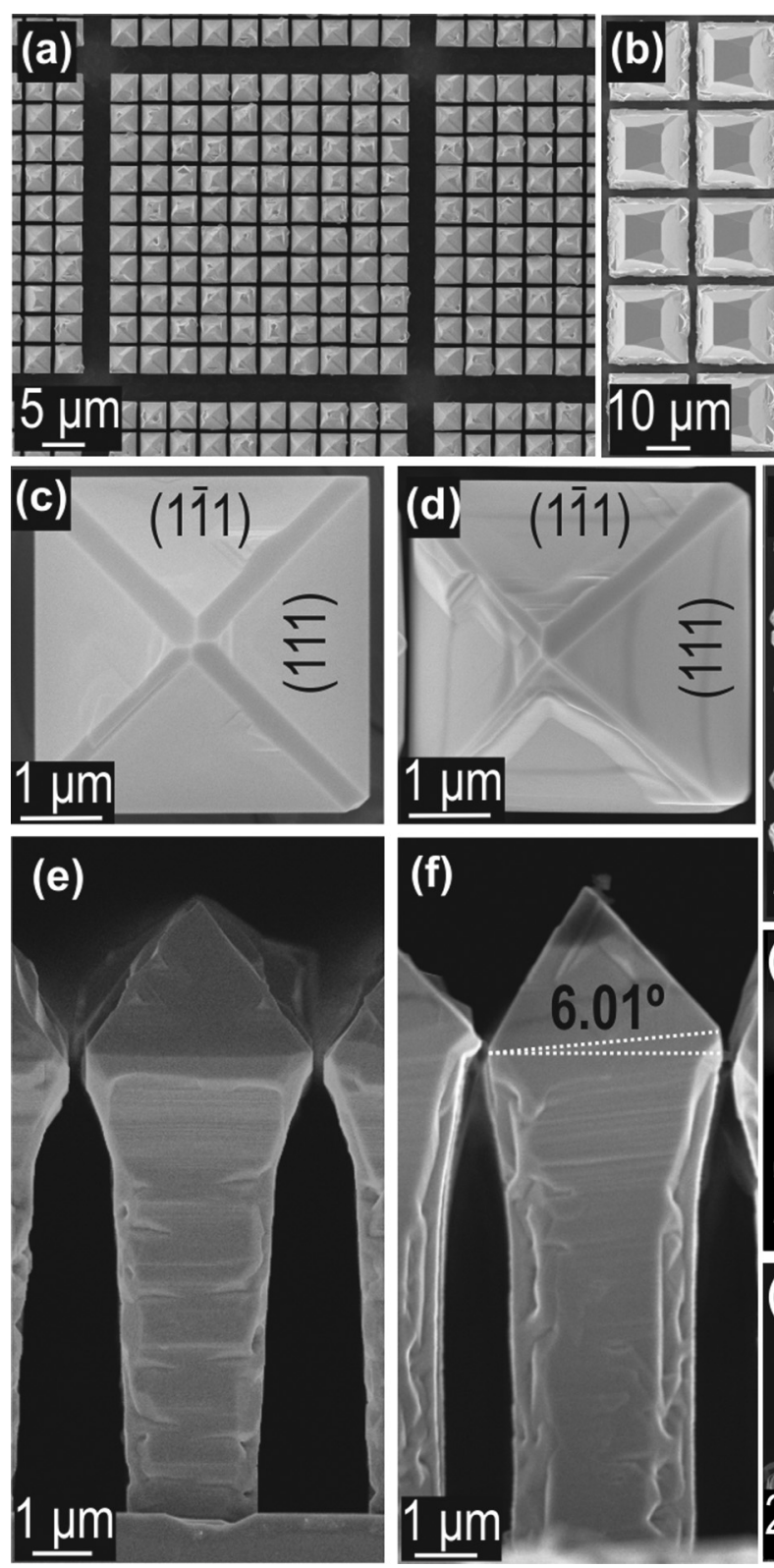
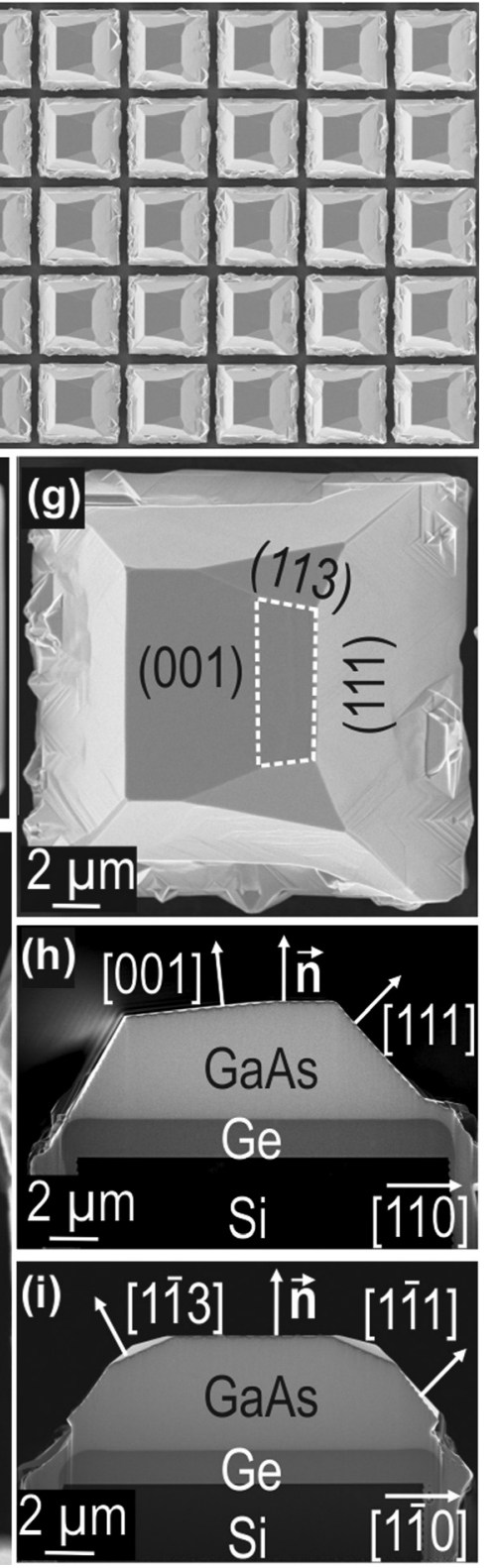

FIG. 1. (a) Top SEM view of GaAs/Ge pyramids grown by MOVPE on top of $8-\mu \mathrm{m}$-tall and $2-\mu \mathrm{m}$-wide Si pillarsetched into $\mathrm{Si}(001)$ substrates offcut $6^{\circ}$ towards [110] and arranged in $10 \times 10$ blocks. (b) Top SEM view of GaAs/Ge crystals on $15-\mu \mathrm{m}$-wide Si pillars separated by $4-\mu \mathrm{m}$-wide trenches. (c) and (d) Detail of the top surface of GaAs pyramids grown on nominal (c) and vicinal (d) Si substrates. The corresponding lateral views (e) and (f) display the tilt of the microstructures grown on offcut substrates. (g) SEM image of the top surface of $4-\mu \mathrm{m}$-tall $\mathrm{GaAs} / \mathrm{Ge}$ crystals grown on $15-\mu \mathrm{m}$ wide Si pillars. In all cases, $2 \mu \mathrm{m}$ of Ge were deposited by LEPECVD prior to the GaAs growth. The FIB cross sections parallel to the (1-10) (h) and (110) (i) planes display the strong morphological asymmetry of $\mathrm{GaAs} / \mathrm{Ge}$ crystals grown on offcut $\mathrm{Si}$ patterned substrates. The direction normal to the $\mathrm{Si}$ offcut surface is indicated by the vector $\vec{n}$. 
The high epitaxial quality of $\mathrm{GaAs} / \mathrm{Ge} / \mathrm{Si}$ material grown by MOVPE was confirmed by HRXRD, both for planar and patterned substrates. The reciprocal space maps (RSM) acquired around the (004) azimuth of GaAs grown on a planar $\mathrm{Ge} / \mathrm{Si}$ substrate and on an array of $9-\mu \mathrm{m}$-wide $\mathrm{Ge}$ coated Si pillars are shown in Figs. 2(a) and 2(b), respectively. The two well separated diffraction peaks found for the planar material correspond to GaAs and Ge (Fig. 2(a)). The symmetric wings along $Q_{x}$ are the result of weak diffuse scattering by the partially relaxed material near thermal cracks. ${ }^{25}$ For the patterned epitaxial material, the diffraction peaks corresponding to $\mathrm{GaAs}$ and $\mathrm{Ge}$ are shifted towards lower $\mathrm{Q}_{\mathrm{z}}$ momenta (Fig. 2(b)), indicating elastic release of the tensile thermal strain. In addition, both diffraction peaks are elongated along the $\mathrm{Q}_{\mathrm{x}}$ direction. This broad diffuse scattering around the $\mathrm{GaAs}(004)$ and $\mathrm{Ge}(004)$ reflections may be related to varying lattice tilts across the diameter of the crystals as a result of elastic thermal strain release. ${ }^{26}$

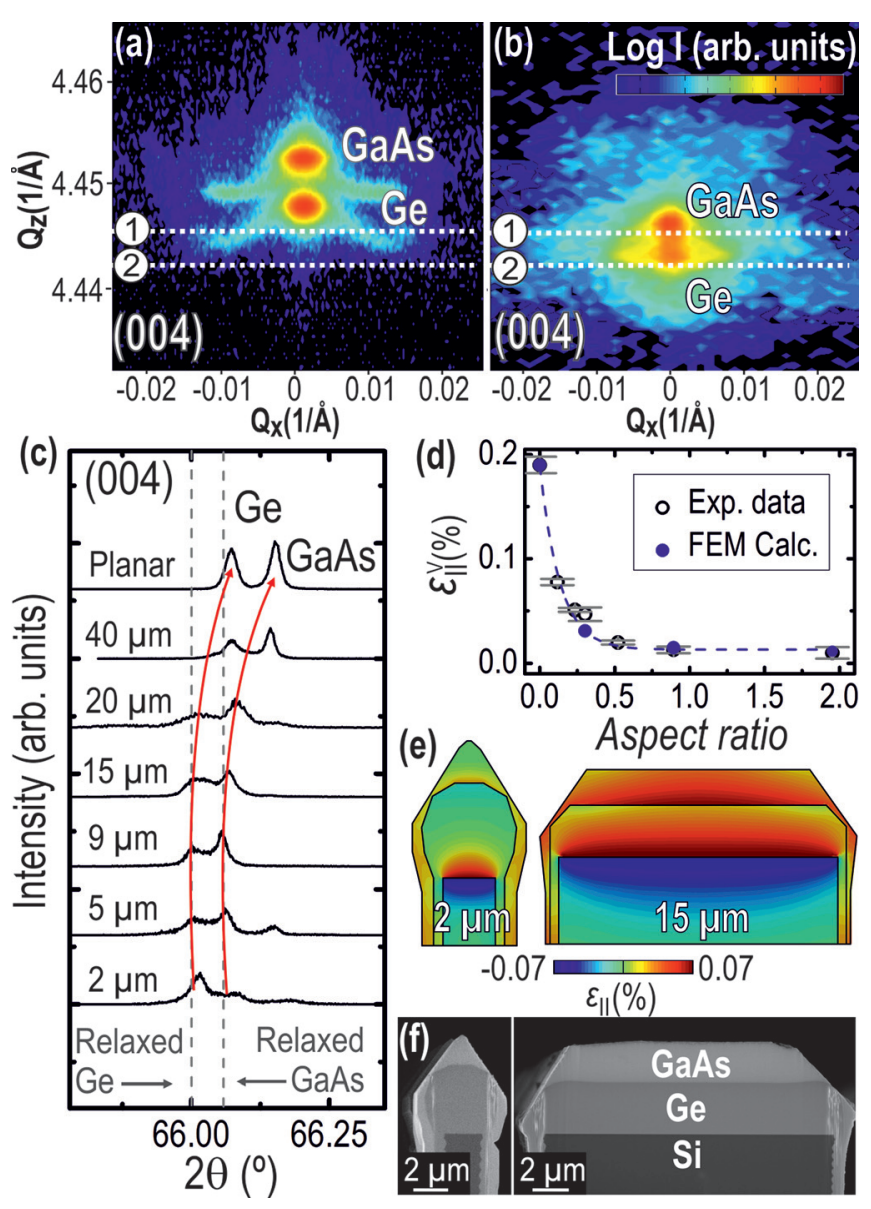

FIG. 2. RSM measured on the (004) azimuth of GaAs grown on a planar $\mathrm{Ge} / \mathrm{Si}$ miscut substrate (a) and on an array of 9 - $\mu \mathrm{m}$-wide $\mathrm{Ge} / \mathrm{Si}$ pillars (b). The $\mathrm{Q}_{\mathrm{z}}$ momenta values for relaxed $\mathrm{GaAs}$ and $\mathrm{Ge}$ are indicated with the dashed lines labeled with 1 and 2, respectively. (c) 2 $\theta-\omega$ XRD scans corresponding to the different $\mathrm{GaAs} / \mathrm{Ge} / \mathrm{Si}$ microstructure sizes (from $2 \mu \mathrm{m}$ to $40 \mu \mathrm{m}$ wide) compared with reference epitaxy grown on a planar $\mathrm{Ge} / \mathrm{Si}$ substrate. The dashed lines indicate the relaxed GaAs and Ge diffraction angles. The red lines are guides to the eye. (d) Strain values for the different $\mathrm{GaAs} / \mathrm{Ge} / \mathrm{Si}$ microstructure sizes compared with the volume strain calculated by FEM. (e) Strain relaxation maps obtained from FEM simulations for GaAs/Ge crystals on 2 and $15 \mu \mathrm{m}$ wide Si pillars. The strain maps in the simulated geometries are compared with their correspondent SEM cross-section images, displayed in (f).

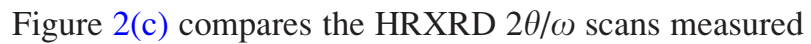
around the (004) reflection for a $\mathrm{GaAs} / \mathrm{Ge}$ layer on a planar $\mathrm{Si}(001)$ substrate and for GaAs/Ge crystals on Si pillars with widths ranging from 2 to $40 \mu \mathrm{m}$. Two intense diffraction peaks corresponding to GaAs and Ge were found for both kinds of substrates. A much weaker component, shifted to higher Bragg angles, may be identified in the lowest two spectra. It stems from the tensile-strained material deposited between the Si pillars. We observe a monotonic shift of the intense peaks towards lower Bragg angles with decreasing $\mathrm{Si}$ pillar size. This shift is again caused by elastic relaxation of the thermal strain in $\mathrm{GaAs} / \mathrm{Ge} / \mathrm{Si}$ heterostructures of high aspect ratio (defined as the ratio between the GaAs/Ge structure height and the Si base width). In order to quantify this relaxation, the HRXRD symmetrical (004) scans were complemented with their corresponding scans for the asymmetrical (224) azimuth. As previously mentioned, the scattering plane was chosen perpendicular to the offcut direction. The step densities along [1-10], the direction perpendicular to the substrate offcut, and along [110], parallel to it, are known to be dissimilar. This leads to different relaxation paths for the epitaxial material, resulting in an additional tilt along $[110] .{ }^{27}$ During the following discussion of the strain relaxation of $\mathrm{GaAs} / \mathrm{Ge} / \mathrm{Si}$ structures we will neglect this anisotropic relaxation and approximate the strain of the epilayers to be biaxial. Up to a Si pillar size of $5 \times 5 \mu \mathrm{m}^{2}$ the Ge and GaAs crystals exhibit an order of magnitude less thermal strain than planar $\mathrm{GaAs} / \mathrm{Ge} / \mathrm{Si}$ material. For structures of $15 \times 15 \mu \mathrm{m}^{2}$, the GaAs strain increases to $\sim 0.05 \%$. A monotonically rising strain was found for increasing structure size up to the value of $0.19 \%$ observed for planar material (Fig. 2(d)).

The strain obtained from HRXRD was compared with the one calculated by the FEM simulations. The strain distribution in the system with the two interfaces was calculated using the thermal strain values of GaAs and Ge layers, obtained from HRXRD on planar areas as initial conditions for a simulated evolution to mechanical equilibrium in the corresponding finite system with free surfaces. The latter ones are treated by the boundary condition $(\vec{\sigma} \cdot \vec{n}=0)$, indicating a stress-free component perpendicular to the surface. The anisotropic elastic constants of GaAs and Ge are used for the internal strain evaluation. ${ }^{28}$ Since the penetration depth of the X-rays is much larger than the height of the crystals investigated in this work (for $\mathrm{Cu} \mathrm{K} \alpha_{1}$ it amounts to several tens of microns for the 004 reflection), the in-plane strains obtained from the FEM calculations had to be averaged over the crystal volume $\left(\varepsilon_{\|}^{\mathrm{V}}\right)$. The corresponding experimental values for planar GaAs and Ge are $\varepsilon_{\|}^{\mathrm{V}}(G a A s)$ $=0.19 \%$ and $\varepsilon_{\|}^{\mathrm{V}}(\mathrm{Ge})=0.14 \%$, respectively. Figure 2(e) shows the calculated in-plane strain maps for the 2 and $15 \mu \mathrm{m}$ wide Si pillars of Fig. 2(f). The described averaging procedure yielded strains of $0.011 \%, 0.015 \%$, and $0.031 \%$ for GaAs on 2, 5, and $15 \mu \mathrm{m}$ wide Si pillars in good agreement with the experimental strains from HRXRD (see Fig. 2(d)).

Figure 3(a) shows the PL spectra of GaAs crystals on 2-40 $\mu \mathrm{m}$ wide Si pillars, compared with the spectra of GaAs layers on planar $\mathrm{Ge} / \mathrm{Si}$ and $\mathrm{GaAs}$ substrates. The PL spectra from the GaAs grown on the various patterned substrates 


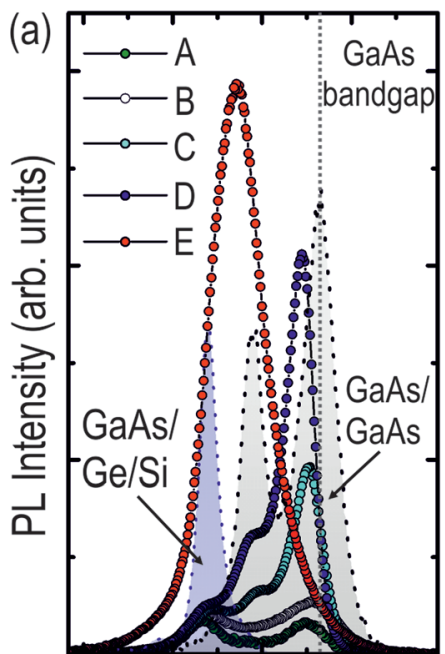

1.441 .471 .501 .53 Energy (eV)

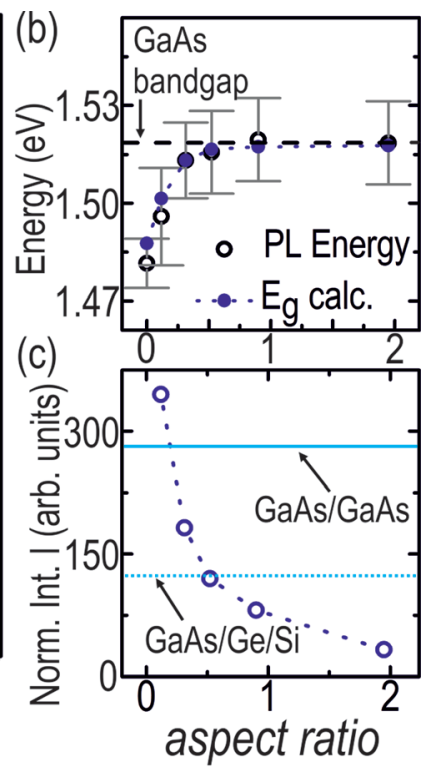

FIG. 3. (a) Low temperature PL spectra of GaAs/Ge microstructures grown on $2 \times 2$ (A), $5 \times 5$ (B), $9 \times 9$ (C), $15 \times 15$ (D) and $20 \times 40$ (E) $\mu \mathrm{m}^{2}$ Si pillars. They are compared with the PL spectrum of a $2 \mu \mathrm{m}$ thick GaAs film grown both on a planar Ge/Si substrate and on a GaAs substrate. (b) Open circles: PL energy obtained from Gaussian fit of the high energy peaks of the luminescence spectra in (a); dashed line and circles: The calculated bandgap shifts using the $\varepsilon_{\|}$measured by HRXRD as an input to Eq. (1). Error bars correspond to the FWHM of the fitted PL band-gap component. (c) Integrated intensity normalized to the absorption volume as a function of the aspect ratio of the Si pillars. The dashed and continuous lines represent the two references, GaAs on a Ge/Si planar substrate and GaAs homoepitaxially grown on GaAs, respectively.

contain three components. For GaAs crystals on $2-\mu$ m-wide $\mathrm{Ge} / \mathrm{Si}$ pillars the most intense line is centered at $1.519 \mathrm{eV}$, in good agreement with band-to-band transition in bulk GaAs. Upon increasing the Si pillar size this line is gradually redshifted, e.g., by about $6 \mathrm{meV}$ for $15-\mu \mathrm{m}$-wide pillars. These small energy shifts can be related to a slight increase of the biaxial tensile strain of the $\mathrm{GaAs} / \mathrm{Ge} / \mathrm{Si}$ system, due to incomplete elastic strain release in the larger structures. The intermediate peak, centered at $\sim 1.498 \mathrm{eV}$ (with small variations for the different sizes) may be assigned to a free electronneutral acceptor transition $\left(\mathrm{e}-\mathrm{C}^{0}, 1.494 \mathrm{eV}\right)$ due to carbon impurities incorporated during the MOVPE growth. ${ }^{15}$ These two lines are present also in the GaAs control epitaxy grown on a semi-insulating GaAs substrate. Finally, the lowest energy optical transition at $1.484 \mathrm{eV}$ coincides with the main line collected from GaAs layers on planar substrates. It can therefore be assigned to emission stemming from the strained material inside the trenches. The PL spectrum collected from the GaAs grown on planar Ge/Si substrates exhibits a double-peak-structure with contributions from GaAs bandgap emission and the e- $\mathrm{C}^{0}$ lines, both strain-shifted by $\sim 30 \mathrm{meV}$ to the red. The strain induced shift $\Delta E$ of the GaAs bandgap $\left(E_{g}\right)$ can be calculated using the expression ${ }^{29}$

$E_{g}=E_{g}^{0}-\Delta E=E_{g}^{0}-\left[-2 a\left(\frac{c_{11}-c_{12}}{c_{11}}\right)+b\left(\frac{c_{11}-2 c_{12}}{c_{11}}\right)\right] \varepsilon_{\|}$,

where $\varepsilon_{\|}$is the biaxial strain, $C_{11}$ and $C_{12}$ are the stiffness coefficients, and $a$ and $b$ are the hydrostatic and shear deformation potentials, respectively, $\left(C_{11}=119 \mathrm{GPa}\right.$, $C_{12}=53.4 \mathrm{GPa}, a=-9.8 \mathrm{eV}$, and $b=-2.0 \mathrm{eV}$ for $\left.\mathrm{GaAs}\right) .^{30}$ A comparison of the PL maxima obtained by Gaussian fits of the different luminescence spectra and the calculated bandgap shifts using the $\varepsilon_{\|}$measured by HRXRD as an input to Eq. (1) can be found in Fig. 3(b). The thermal strain induced during cooling from the HRXRD measurement temperature to the $\mathrm{PL}$ characterization temperature $(\mathrm{T}=5 \mathrm{~K})$ was taken into account. The good agreement between the calculated and the observed PL peak positions confirms the previously discussed size dependent elastic strain relaxation of $\mathrm{GaAs}$ crystals grown on patterned $\mathrm{Ge} / \mathrm{Si}$.

Figure 3(c) shows the dependence of the integrated PL intensity on the aspect ratio of the various patterns. The PL contributions of the GaAs bandgap and e- $\mathrm{C}^{0}$ lines were deconvoluted from the PL generated on the strained material in the trenches by Gaussian fitting. We first determined the absorption length $\mathrm{L}$ at the PL excitation wavelength. Then, we calculated the corresponding absorption volumes from $\mathrm{L}$ and the dimensions of the GaAs crystals extracted from plan-view and cross-section SEM images. Finally, the PL intensities were normalized to these absorption volumes and compared with the one of a planar GaAs layer.

The normalized PL intensity was found to increase with increasing pillar size. This increase may be related to the larger proportion of (001) and offcut (001) surfaces compared to $\{111\}$ facets in the larger GaAs crystals (Fig. 1). First, GaAs crystals on small pillars are mainly composed of $\{111\}$ facets, a large part of which is in close proximity to the Ge mesas. This may lead to enhanced carrier diffusion towards the deep electronic levels present at the GaAs-Ge interface where excitons recombine non-radiatively. Second, compared with the (001) surfaces, the PL collected from tilted $\{111\}$ planes is largely affected by Fresnel losses and total internal reflection reducing its efficiency for small GaAs crystals with their higher proportion of slanting facets. The integrated PL intensity of the GaAs crystals on $20 \times 40 \mu \mathrm{m}^{2}$ Si pillars is comparable with the one collected from the GaAs on GaAs reference. Moreover, a factor 5 increase was found when comparing this pattern with respect to GaAs layers on planar Ge/Si substrates. This increase can only be explained by a substantially improved GaAs epitaxial quality on patterned substrates compared with planar material.

Spatially resolved micro-PL spectra of single GaAs crystals were recorded by scanning a diffraction limited spot across the structures in a confocal arrangement. Figure 4(a) shows a logarithmic intensity map obtained by integrating the near band-gap emission in a $20 \times 20 \mu \mathrm{m}^{2}$ area containing four $\mathrm{GaAs} / \mathrm{Ge}$ crystals on $9 \times 9 \mu \mathrm{m}^{2} \mathrm{Si}$ pillars. The sub-micron optical resolution allows us to match the observed luminescence pattern with the GaAs crystal morphology. A representative case of the morphology of such an area is depicted in the SEM image of Fig. 4(b). The correspondence between the crystal morphology and the PL maps were established by mapping the laser reflection in the same area. The spots labeled with capital letters (A-F) in both Figs. 4(a) and 4(b) correspond to the micro-PL spectra presented in Fig. 4(c). The luminescence collected from the top (001) facet and offcut (001) surface (spectra B, C, D, and E 


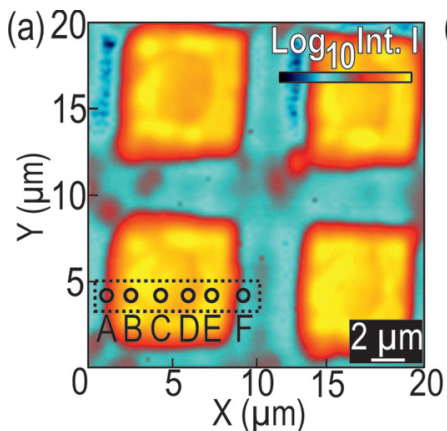

(b)
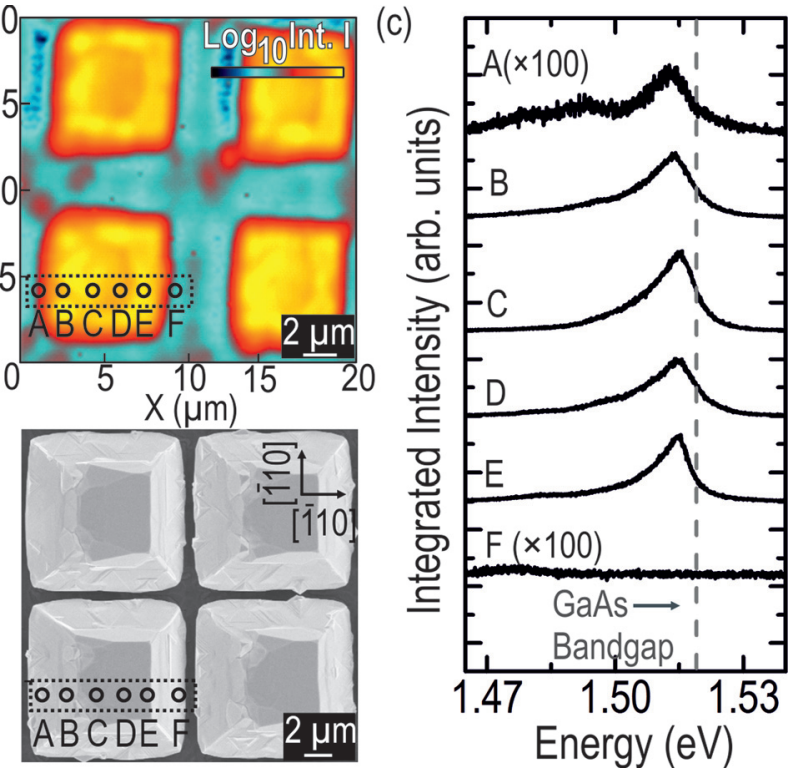

FIG. 4. (a) Micro-PL integrated intensity map of a $20 \times 20 \mu \mathrm{m}^{2}$ area containing four GaAs crystals grown on Ge coated $9 \times 9 \mu \mathrm{m}^{2}$ Si pillars. A representative case of such an area is depicted in the SEM image in Figure (b). (c) Micro-PL spectra collected from different points of a GaAs crystal. The spatial positions of the corresponding spectra and their integrated intensities are indicated in Figs. 4(a) and 4(b), respectively.

from Figure 4(c)) contains two main lines, the previously mentioned free electron-neutral acceptor transition $\left(e-\mathrm{C}^{0}\right)$ and the broadened GaAs band edge emission. Both lines are red-shifted by $\sim 3 \mathrm{meV}$, in good agreement with the value expected from the discussion of Fig. 3(b). The broad asymmetric tail towards low energies of the GaAs band edge emission (FWHM $=9 \mathrm{meV}$ ) can be tentatively attributed to the presence of doping impurities in the GaAs matrix. The doping concentration estimated from the FWHM value, $\mathrm{n} \approx 1.2 \times 10^{17} \mathrm{~cm}^{-3}$, suggests strong Ge diffusion during the initial stages of the GaAs growth on the Ge coated $\mathrm{Si}$ pillars. We also observe a strong decrease of the micro-PL intensity at the positions corresponding to the $\{111\}$ facets (A and F), which supports the interpretation that the dependence of the spatially averaged PL intensity on crystal size (Fig. 3(c)) may be caused by the low PL efficiency at slanting facets.

In summary, mask-less integration of high quality epitaxial GaAs on patterned $\mathrm{Ge} / \mathrm{Si}$ substrates by MOVPE was demonstrated. With decreasing size the GaAs crystals evolve towards strain-free pyramidal morphologies formed by energetically stable $\{111\}$ facets. When grown on misoriented substrates they exhibit a rotation of their growth axis towards the natural directions of GaAs. The ability to achieve well oriented $\mathrm{GaAs}(001)$ surfaces from the misoriented Si substrates required for APD prevention is of utmost interest, as it allows to avoid the higher roughness common to miscut surfaces. Quantum wells with low interface step densities incorporated into such GaAs crystals are therefore expected to exhibit much higher mobilities due to decreased interface roughness scattering. We therefore consider these results to be a step forward towards the monolithic hetero-integration of III-V based micro- and optoelectronic devices on silicon.

Financial support by the Swiss Federal Program NanoTera through projects NEXRAY and COSMICMOS and Spanish MINECO and CAM through projects EPICNANOTICS and Q\&C Light are gratefully acknowledged. We acknowledge K. Mattenberger for technical support and B. Batlogg, J. Fompeyrine, E. Gini, and M. Meduna for valuable scientific discussions; FIRST center for micro- and nanoscience and EMEZ electron microscopy center at ETH Zürich for making available its infrastructures, and Pilegrowth Tech Srl for providing their technical expertise.

${ }^{1}$ S. Lourdudoss, Curr. Opin. Solid State Mater. Sci. 16, 91 (2012).

${ }^{2}$ M. Akiyama, Y. Kawarada, and K. Kaminishi, J. Cryst. Growth 68, 21 (1984).

${ }^{3}$ G. Brammertz, Y. Mols, S. Degroote, V. Motsnyi, M. Leys, G. Borghs, and M. Caymax, J. Appl. Phys. 99, 093514 (2006).

${ }^{4}$ K. Nozawa and Y. Horikoshi, Jpn. J. Appl. Phys., Part 2 29, L540 (1990).

${ }^{5}$ R. Fischer, N. Chand, W. Kopp, H. Morkog, L. P. Erickson, and R. Youngman, Appl. Phys. Lett. 47, 397 (1985).

${ }^{6}$ Y. González, L. González, and F. Briones, Jpn. J. Appl. Phys., Part 2 31, L816 (1992).

${ }^{7}$ S. M. Ting and E. A. Fitzgerald, J. Appl. Phys. 87, 2618 (2000).

${ }^{8}$ J. A. Carlin, S. A. Ringel, E. A. Fitzgerald, M. Bulsara, and B. M. Keyes, Appl. Phys. Lett. 76, 1884 (2000).

${ }^{9}$ R. Ginige, B. Corbett, M. Modreanu, C. Barrett, J. Hilgarth, G. Isella, D. Chrastina, and H. von Känel, Semicond. Sci. Technol. 21, 775 (2006).

${ }^{10}$ M. Richter, E. Uccelli, A. G. Taboada, D. Caimi, N. Daix, M. Sousa, C. Marchiori, H. Siegwart, C. V. Falub, H. von Känel, F. Isa, G. Isella, A. Pezous, A. Dommannd, P. Niedermann, and J. Fompeyrine, J. Cryst. Growth 378, 109 (2013).

${ }^{11}$ S. Bietti, C. Somaschini, S. Sanguinetti, N. Koguchi, G. Isella, and D. Chrastina, Appl. Phys. Lett. 95, 241102 (2009).

${ }^{12}$ Y. Komatsu, K. Hosotani, T. Fuyuki, and H. Matsunami, Jpn. J. Appl. Phys., Part 1 36, 5425 (1997).

${ }^{13}$ E. A. Fitzgerald and N. Chand, J. Electron. Mater. 20, 839 (1991).

${ }^{14}$ G. Vanamu, A. K. Datye, R. Dawson, and S. H. Zaidi, Appl. Phys. Lett. 88, 251909 (2006).

${ }^{15}$ J. Z. Li, J. Bai, J. M. Hydrick, J. S. Park, C. Major, M. Carroll, J. G. Fiorenza, and A. Lochtefeld, J. Cryst. Growth 311, 3133 (2009).

${ }^{16}$ G. Brammertz, Y. Mols, S. Degroote, J. V. Steenbergen, M. Leys, G. Borghs, and M. Caymax, J. Cryst. Growth 297, 204 (2006).

${ }^{17}$ A. Marzegalli, F. Isa, H. Groiss, E. Müller, C. V. Falub, A. G. Taboada, P. Niedermann, G. Isella, F. Schäffler, F. Montalenti, H. von Känel, and L. Miglio, Adv. Mater. 25(32) 4408 (2013).

${ }^{18}$ R. Bergamaschini, F. Isa, C. V. Falub, P. Niedermann, E. Müller, G. Isella, H. von Känel, and L. Miglio, Surf. Sci. Rep. 68, 390 (2013).

${ }^{19}$ C. V. Falub, H. von Känel, F. Isa, R. Bergamaschini, A. Marzegalli, D. Chrastina, G. Isella, E. Müller, P. Niedermann, and L. Miglio, Science 335, 1330 (2012).

${ }^{20}$ F. Laermer and A. Schilp, U.S. patent 5,501,893 (26 March 1996).

${ }^{21}$ C. Rosenblad, H. von Känel, M. Kummer, A. Domman, and E. Muller, Appl. Phys. Lett. 76, 427 (2000).

${ }^{22}$ W. I. Wang, Appl. Phys. Lett. 44, 1149 (1984).

${ }^{23}$ V. K. Yang, M. Groenert, C. W. Leitz, A. J. Pitera, M. T. Currie, and E. A. Fitzgerald, J. Appl. Phys. 93, 3859 (2003).

${ }^{24}$ O. Kayser, J. Cryst. Growth 107, 989 (1991).

${ }^{25}$ D. Colombo, E. Grilli, M. Guzzi, S. Sanguinetti, S. Marchionna, M. Bonfanti, A. Fedorov, H. von Känel, G. Isella, and E. Müller, J. Appl. Phys. 101, 103519 (2007).

${ }^{26}$ C. V. Falub, M. Meduňa, D. Chrastina, F. Isa, A. Marzegalli, T. Kreiliger, A. G. Taboada, G. Isella, L. Miglio, A. Dommann, and H. von Känel, Sci. Rep. 3, 2276 (2013).

${ }^{27}$ H. Nagai, J. Appl. Phys. 45, 3789 (1974).

${ }^{28}$ L. D. Landau and E. M. Lifshitz, Theory of Elasticity (Pergamon Press, Oxford, 1970), Vol. 7.

${ }^{29}$ C. Van de Walle, Phys. Rev. B 39, 1871 (1989).

${ }^{30}$ W. Stolz, F. E. G. Guimaraes, and K. Ploog, J. Appl. Phys. 63, 492 (1988). 